\title{
Anti-depressant-like effects of Jieyu chufan capsules in a mouse model of unpredictable chronic mild stress
}

\author{
HUI ZHAO ${ }^{1-4}$, BINGYU LIANG ${ }^{2,5}$, LINJIE YU ${ }^{1-4}$ and YUN XU ${ }^{1-4}$ \\ ${ }^{1}$ Department of Neurology, Affiliated Drum Tower Hospital, Nanjing University Medical School; \\ ${ }^{2}$ State Key Laboratory of Pharmaceutical Biotechnology, Nanjing University; ${ }^{3}$ Jiangsu Key Laboratory \\ for Molecular Medicine, Nanjing University Medical School; ${ }^{4}$ Department of Neurology, Nanjing Clinic \\ Medicine Center for Neurological and Psychiatric Diseases; ${ }^{5}$ Department of Neurology, Affiliated Drum \\ Tower Hospital, Nanjing University of Chinese Medicine, Nanjing, Jiangsu 210008, P.R. China
}

Received April 25, 2016; Accepted March 24, 2017

DOI: $10.3892 /$ etm.2017.4601

\begin{abstract}
Jieyu chufan (JYCF) is a well-known Chinese traditional medicine used for depression; however, the molecular mechanism underlying its anti-depressant action has remained elusive. In the present study, the anti-depressant effects of JYCF and the potential mechanisms were investigated in a mouse model. Five groups of 12 C57BL/6 mice each were used in the study, including a normal control group (NC group), a model control group (MC group) and three groups, which received different doses of JYCF $(1.25,2.5$ and $5 \mathrm{~g} / \mathrm{kg})$ orally for 21 days (JYCF groups). The MC group and the three JYCF groups were subjected to 3 weeks of unpredictable chronic mild stress (UCMS) to induce depression-like behavior. All groups were subjected to a sucrose consumption test along with a forced swimming test to confirm depression-like behavior, an open-field test and an elevated plus maze test to confirm anxiety-like behavior, and a Morris water maze test to evaluate spatial learning and memory. In addition, synaptic density in the hippocampus was evaluated and western blot and immunostaining were used to analyze hippocampal expression of postsynaptic density protein-95 (PSD95), synaptophysin (Syn), cyclic adenosine monophosphate response element binding protein (CREB), brain-derived neurotrophic factor (BDNF), Akt and glycogen synthase kinase (GSK)-3 $\beta$ as well as their phosphorylated (p) versions. The results showed that JYCF (2.5 and $5 \mathrm{~g} / \mathrm{kg}$ ) alleviated depressive-like behaviors and increased synaptic density in UCMS mice. Moreover, JYCF upregulated the expression of PSD95, Syn and BDNF and increased phosphorylated Akt, CREB and GSK-3 $\beta$ in the hippocampus. These results suggested that JYCF exerts an
\end{abstract}

Correspondence to: Professor Yun Xu, Department of Neurology, Affiliated Drum Tower Hospital, Nanjing University Medical School, 321 Zhongshan Road, Nanjing, Jiangsu 210008, P.R. China E-mail: xuyun20042001@aliyun.com

Key words: Chinese traditional medicine, Jieyu chufan capsules, depression, unpredictable chronic mild stress anti-depressant-like activity in UCMS-induced mice, which is likely to be mediated by reversing the stress-induced disruption of BDNF and GSK-3 $\beta$ activity.

\section{Introduction}

Depression is a common psychiatric disorder characterized by a persistent low mood, feeling of helplessness and suicidal tendencies, which causes enormous personal suffering and economic loss, as well as sluggish thought patterns and cognitive function (1). As the underlying mechanisms of depression are relatively complex and are not explicit, currently available treatments continue to have significant limitations, including low response rates and frequent remission, treatment resistance, severe adverse effects and a delayed clinical response (weeks to months) (2). Therefore, it is important to probe and develop more effective anti-depressant medications. It has been reported that depression is associated with neuronal atrophy and loss, including reduction in the number of spine synapses, one of the key points of connection between neurons (3). The leading hypothesis on depression suggests that neuronal plasticity and impaired synaptic function are critical for mediating behavioral responses to anti-depressants $(4,5)$. Studies have indicated that the impairment of synaptic plasticity in the hippocampus may be a core factor in the pathophysiology of depression $(6,7)$.

Brain-derived neurotrophic factor (BDNF) is the key regulator of neuronal plasticity and strongly influences synaptogenesis, spine formation, neuronal survival and adult hippocampal neurogenesis (8-10). There is growing evidence that links BDNF to depression; post-mortem analyses of depressive patients have found a reduction of BDNF in brain and serum $(11,12)$. Decreased BDNF mRNA expression in the hippocampus contributes to depression-like behavior in rats (13), while brain infusion of BDNF in animals produced anti-depressant-like effects (14). The glycogen synthase kinase-3 (GSK-3) pathway is thought to have a principal role in long-term depression and long-term potentiation in the hippocampus (15). In addition, studies including behavioral assessments indicated that GSK-3 $\beta$ dysregulation promotes mood disorders (16). The classical mood stabilizer lithium is a 
direct inhibitor of GSK-3 (17). Pre-clinical studies on animal models have shown anti-depressant-like effects of GSK-3 $\beta$ inhibitors $(18,19)$. These results suggested that regulating the stimulation of BDNF and suppression of GSK-3 $\beta$ may be a potential approach for treating depression.

Traditional Chinese Medicine is gaining attention for its mild nature, its tendency to maintain an individual balance and its multiple target effect (20). Jieyu chufan capsules (JYCF) consist of four Chinese herbs: Gardenia jasminoides Ellis (Zhi-Zi), Forsythia suspensa Vahl (Lian-Qiao), Cortex Magnolia officinalis (Hou-Pu) and rhizoma Pinelliae ternatae (Ban-Xia). They are a novel Chinese herbal medicine for the treatment of depression. The JYCF formula is derived from the zhi-zi-hou-pu and ban-xia-hou-pu decoctions. The first description of these two decoctions was recorded in Shan-han-lun and Jin-gui-yao-lue written by Zhang Zhongjing during the Han Dynasty (150-219 A.D.) and they have historically been used to relieve depression symptoms, anxiety disorders, obsessive-compulsive disorders and hysteria (21-23). Gardenia jasminoides Ellis is the major active ingredient of JYCF, but the molecular mechanism underlying these decoctions has remained elusive.

In the present study, the anti-depressant effects of JYCF were first assessed in a mouse model of unpredictable chronic mild stress (UCMS)-induced depression. Evidence was provided that JYCF suppressed the impairment of synaptic density by regulating BDNF and GSK-3 $\beta$ activity, suggesting that JYCF may potentially be developed into a drug for the treatment of depression.

\section{Materials and methods}

Ethics statement. All animal care and experimental procedures complied with the National Institute of Health Guidelines for the Care and Use of Laboratory Animals. All procedures that involved animals were in compliance with the European Community Council Directive of November 24, 1986 and approval was granted by the Ethics Committee of Nanjing Drum Tower Hospital (Nanjing, China; permission no. 2014-085-01). All surgical procedures were performed under sodium pentobarbital anesthesia and all efforts were made to reduce suffering.

Animals and groups. A total of 60 healthy adult female C57BL/6 mice (age, 4-5 weeks; weight, 18-21 g), were provided by the Animal Experiment Center of Nanjing Drum Tower Hospital (the Affiliated Hospital of Nanjing University Medical School; Nanjing, China). The animals were kept in the laboratory ( 5 per cage) for 2 weeks prior to experimentation, with free access to food and water. The animal room had a temperature of $23 \pm 1.5^{\circ} \mathrm{C}$ with $50 \%$ relative humidity and a 12-h light/dark cycle (light on from 7:00 am to 7:00 pm). The behavioral experiments were performed during the light phase. Mice were randomly divided into five groups with 12 mice in each group including: Normal control group (NC group), model control group (MC group), JYCF $1.25 \mathrm{~g} / \mathrm{kg}$ group, JYCF $2.5 \mathrm{~g}$ crude drug/kg group and JYCF $5 \mathrm{~g}$ crude $\mathrm{drug} / \mathrm{kg}$ group (JYCF groups).

Drugs and reagents. JYCF powder was provided by Shijiazhuang Yiling Pharmaceutical Factory (Shijiazhuang,
China). All medicinal components of JYCF were ground to superfine $(\leq 10 \mu \mathrm{m})$ powder by a micronizer and prepared as 0.38-g capsules. The NC and MC groups received no medication, while JYCF was administered at $1.25,2.5$ or $5 \mathrm{~g} / \mathrm{kg}$ to the three JYCF groups. The outer coating of the capsule was removed, the drugs were dissolved in distilled water and intragastrically administered at $10 \mathrm{ml} / \mathrm{kg}$. At the same time, mice in the $\mathrm{NC}$ and $\mathrm{MC}$ groups were given $10 \mathrm{ml} / \mathrm{kg}$ distilled water. JYCF or water was administered $1 \mathrm{~h}$ prior to each behavioral test to avoid potential stress induced by gavage. Administration was continuous during the entire three weeks.

Establishment of the UCMS model. The UCMS model was established following a modified Willner's method $(24,25)$. Mice of the NC group were treated normally, while mice in the other four groups were subjected to UCMS daily between 11:00 am and 12:00 pm for 3 successive weeks.

Mice were housed individually in cages and exposed to the following stressors: Food or water deprivation (24 h), damp sawdust $(12 \mathrm{~h})$, forced swimming in cold water $\left(4^{\circ} \mathrm{C}\right)$ for $4 \mathrm{~min}$, tail suspension (5 min), inversion of light/dark cycle $(24 \mathrm{~h})$, cage tilting $\left(45^{\circ} \mathrm{C}, 12 \mathrm{~h}\right)$ and tail nipping (10 times for $5 \mathrm{sec}$ per time). To prevent familiarization and provide an unpredictable feature, all stressors were randomly scheduled over a 1-week period and repeated throughout the 3 -week experiment.

Sucrose preference test. The sucrose preference test was used for evaluating depression-like behavior and was performed as described in previous studies $(26,27)$. Prior to the test, all mice were given a free choice between two bottles-one with $1 \%$ sucrose solution and another with tap water-over $24 \mathrm{~h}$. To prevent potential location preference, the position of the bottles was changed after $12 \mathrm{~h}$. Mice were deprived of food and water for $24 \mathrm{~h}$ prior to the test. Bottles were weighed prior to each test and then re-weighed one hour later. The sucrose intake was measured by comparing the weight of the bottles prior to and after the test.

Forced swimming test. A forced swimming test was used for evaluating depression-like behavior. A modified protocol was used as described by Porsolt et al (28). In brief, 30 min after JYCF injection, mice were forced to individually swim in a glass cylinder (diameter, $13 \mathrm{~cm}$; height, $19 \mathrm{~cm}$ ) filled with water $\left(25 \pm 1^{\circ} \mathrm{C}\right)$ at a depth of $10 \mathrm{~cm}$. The water was changed after each trial. All animals were forced to swim for $5 \mathrm{~min}$ and the immobility time (including passive swimming) during the test was recorded. Immobilization and passive swimming were defined as the mouse floating in the water without struggling, making only those movements necessary to keep its head above the water. Observers were blinded to grouping of mice.

Open field test. An open field test was used for evaluating emotional and ambulatory behavior. Apparatus and methods used were similar to those previously described (29). Mice were placed individually in the dark in a wooden box $(100 \times 100 \times 40 \mathrm{~cm})$ with the floor divided into $25(5 \times 5)$ squares. The apparatus was illuminated with a red bulb $(50 \mathrm{~W})$ on the ceiling. In each test, mice were placed in the central area and allowed to explore freely for $5 \mathrm{~min}$. During the test time, the 


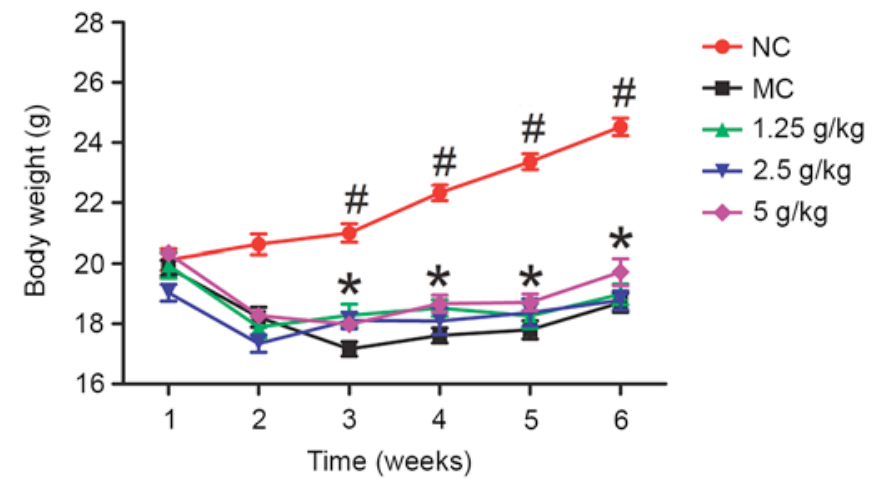

Figure 1. Body weight in NC, MC and JYCF groups was determined every week for 6 consecutive weeks. In the week 3, body weight of MC and NC group mice significantly deceased. NC group mice consecutively decreased in the week 6. ${ }^{*} \mathrm{P}<0.05,{ }^{\#} \mathrm{P}<0.01$ compared with $\mathrm{MC}$ group. $\mathrm{NC}$, negative control; MC, model control group; $1.25 / 2.5 / 5 \mathrm{~g} / \mathrm{kg}$, model mice treated with 1.25/2.5/5 g/kg JYCF; JYCF, Jieyu chufan.

number of crossings (squares crossed with all paws) and rearings (raising of front paws) was recorded. The open field arena was thoroughly cleaned between tests. The observers were blinded to the treatment of the mice.

Elevated plus maze test. The elevated plus maze test was used for evaluating anxiety-associated behavior (30). The apparatus consisted of two opposing open arms $(45 \times 10 \mathrm{~cm})$ and two closed arms $(45 \times 10 \times 38 \mathrm{~cm})$ that extend from a central platform $(10 \times 10 \mathrm{~cm})$, elevated $50 \mathrm{~cm}$ above the floor. During testing, the mice were placed individually on the central platform facing the open arms of the maze and allowed to explore the maze for $5 \mathrm{~min}$. Behavior was monitored using a video camera and analyzed with a computerized tracking system (Ethovision 3.1.16; Noldus IT, Wageningen, The Netherlands). Time spent in the open and closed arms (and their edges) was recorded. The anxiety level was measured by the time spent in the open arm. The observer was blinded to group classification to avoid bias.

Morris water maze (MWM) test. The MWM test was used for evaluating spatial learning and memory and was performed as previously described (31). In brief, during the acquisition phase trials (days 1-5), mice were allowed to stay on the platform for $5 \mathrm{sec}$ if they were able to find it within $60 \mathrm{sec}$. If they could not, $60 \mathrm{sec}$ was regarded as the escape latency and mice were allowed to remain on the platform for $20 \mathrm{sec}$ to memorize the platform location. During the probe trial phase, after removing the platform, mice were allowed to swim for $60 \mathrm{sec}$ for two tests. The test was performed in four training trials per day for six consecutive days. All movements were recorded by a computerized video system (ANY-maze; Stoelting, Wood Dale, IL, USA). In order to exclude the influence of artificial factors, the MWM test was performed by two observers who were blinded to the group assignments of the mice.

Tissue preparation. After behavioral tests, mice were deeply anesthetized with intraperitoneal injection of sodium pentobarbital (50 mg/kg body weight). Bilateral hippocampi were rapidly dissected and homogenized in lysis buffer (Beyotime Institute of Biotechnology, Inc., Haimen, China), centrifuged at $12,000 \times \mathrm{g}$ for $30 \mathrm{~min}$ at $4^{\circ} \mathrm{C}$ and the supernatants were collected for western blot assay. Whole mouse brains were removed, washed with $1 \mathrm{X}$ phosphate-buffered saline and prepared for Golgi silver staining.

Western blot analysis. Total protein was estimated using a BCA Protein Quantification kit (Bioworld Technology Inc., St. Louis Park, MN, USA). Samples were then mixed with 5X loading buffer and boiled for $5 \mathrm{~min}$. Equal amounts of protein extract $(30 \mu \mathrm{g})$ were separated by $10 \%$ SDS/PAGE and then transferred onto polyvinylidene difluoride membranes. After being blocking with $5 \%$ powdered skimmed milk for $1.5 \mathrm{~h}$, membranes were incubated overnight at $4^{\circ} \mathrm{C}$ with primary antibodies to GSK-3 $\beta$ (cat. no. 12,456), phosphorylated (p)-GSK-3 $\beta$ (cat. no. 5,558) or postsynaptic density protein-95 (PSD-95; cat. no. 3450; all 1:1,000 dilution), p-cyclic adenosine monophosphate response element binding protein (p-CREB; cat. no. 9,198) or Synaptophysin (Syn; cat. no. 5,461; 1:500 dilution), CREB (cat. no. 9,197; 1:500 dilution), p-AKT, (cat. no. 4,060; 1:500 dilution), AKT (all Cell Signaling Technology, Inc., Danvers, MA, USA; cat. no. 9,272; 1:1,000 dilution) or $\beta$-tubulin (Bioworld Technology, Inc.; cat. no. bs1482; 1:5,000 dilution), or BDNF (Epitomics; Abcam, Cambridge, UK; cat. no. 3160-1; 1:500 dilution). Subsequently, membranes were treated with horseradish peroxidase-conjugated secondary antibodies (Bioworld Technology, Inc.; cat. no. bs13278; 1:5,000 dilution) and incubated for $2 \mathrm{~h}$ at room temperature. The blots were visualized with chemiluminescence reagents using an ECL kit (Bioworld Technology, Inc.). Optical density of the bands was determined using Quantity One Software (4.62; Bio-Rad Laboratories, Inc., Hercules, CA, USA).

Golgi staining. Golgi staining was performed following the manufacturer's instructions (FD Rapid GolgiStain Kit; FD NEurotexhnologies, Inc., Ellicott City, MD, USA). In brief, whole brains were impregnated with silver solution for 2 weeks and then sectioned at $100 \mu \mathrm{m}$ on a cryostat. Sections were mounted on adhesive microscope slides and air-dried at room temperature in the dark. For synaptic density quantification, the number of synapses was counted along dendritic segments of equivalent length from the CA1 region of the hippocampi.

Statistical analysis. All statistical analysis was performed using SPSS 16.0 software (SPSS Inc., Chicago, IL, USA). Values are expressed as the mean \pm standard error of the mean. Differences between mean values were evaluated using one-way analysis of variance with post-hoc tests. $\mathrm{P}<0.05$ was considered to indicate a statistically significant difference.

\section{Results}

Effects of JYCF on body weight. Fig. 1 shows the body weight in the five groups during the experimental period. After week 1 , there were no significant differences between the five groups ( $\mathrm{P}>0.05)$. After 6 weeks, the body weight in the MC group was significantly lower than that in the NC group $(\mathrm{P}<0.01)$ and in the $5 \mathrm{~g} / \mathrm{kg}$ JYCF group $(\mathrm{P}<0.05)$.

JYCF affects depression-like behavior in UCMS mice. Fig. 2A shows that the sucrose preference rate in the MC group was 


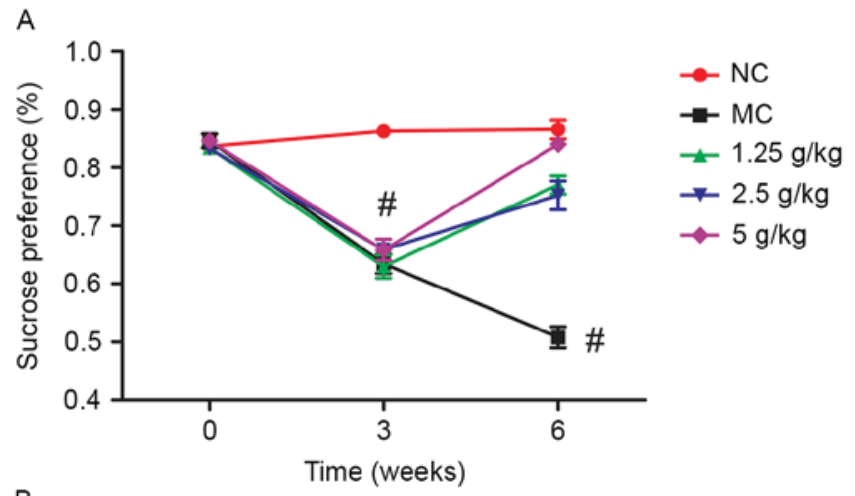

B

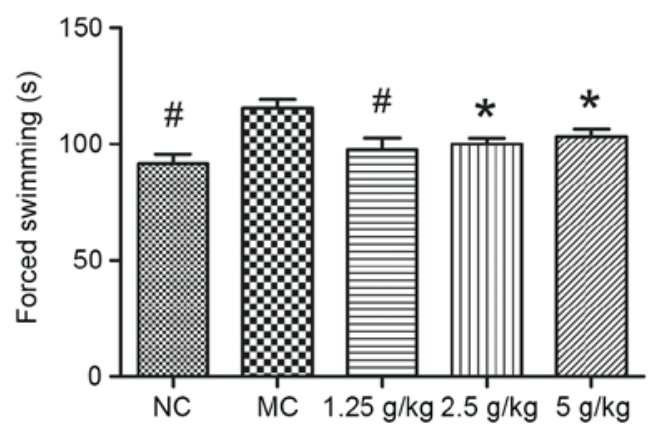

Figure 2.JYCF affected depression-like behavior in UCMS mice. (A) Sucrose intake of NC, MC and JYCF groups was determined at weeks 1, 3 and 6. (B) Total immobilized time was measured using a forced swimming test at week 6 after final UCMS exposure. Values are expressed as the mean \pm standard error of the mean of three independent experiments. ${ }^{\text {}} \mathrm{P}<0.05,{ }^{~} \mathrm{P}<0.01$ compared with MC group. JYCF, Jieyu chufan; NC, negative control; MC, model control group; $1.25 / 2.5 / 5 \mathrm{~g} / \mathrm{kg}$, model mice treated with $1.25 / 2.5 / 5 \mathrm{~g} / \mathrm{kg}$ JYCF; JYCF, Jieyu chufan; UCMS, unpredictable chronic mild stress.

lower than that in the $\mathrm{NC}$ group $(\mathrm{P}<0.01)$. Compared with the MC group, after regular treatment with JYCF, the sucrose intake rates in UCMS-exposed mice were all increased $(\mathrm{P}<0.01)$.

Fig. 2B shows the results of the forced swimming tests. Compared with the NC group, the total immobilized time in the MC group was significantly longer compared with that in the other groups $(\mathrm{P}<0.05)$. After regular treatment with JYCF, the total immobilized time in the UCMS-exposed mice was significantly reduced $(1.25 \mathrm{~g} / \mathrm{kg}, \mathrm{P}<0.01 ; 2.5$ and $5 \mathrm{~g} / \mathrm{kg}, \mathrm{P}<0.05)$.

JYCF affects anxiety-like behavior in UCMS mice. Fig. 3 shows the results of the open-field test. Compared with the NC group, the number of square crossings and rearings in the MC group was significantly decreased, which was significantly inhibited by treatment with 2.5 and $5 \mathrm{~g} / \mathrm{kg} \mathrm{JYCF}$ $(\mathrm{P}<0.05)$. However, there were no differences in the number of square crossings and rearings between the $\mathrm{MC}$ group and the $1.25 \mathrm{~g} / \mathrm{kg}$ JYCF group ( $\mathrm{P}>0.05$; Fig. $3 \mathrm{~A}$ and $\mathrm{B}$ ).

Fig. 4 shows the results of the elevated plus maze test. Compared with those in the $\mathrm{NC}$ group, mice in the $\mathrm{MC}$ group spent significantly less time in the open arm and more time in the closed arm, which was reversed by treatment with JYCF at 2.5 and $5 \mathrm{~g} / \mathrm{kg}(\mathrm{P}<0.05$; Fig. $4 \mathrm{~A}$ and $\mathrm{B})$. The number of entries in the open arms in the MC group was also significantly decreased compared with that in the NC group, which was reversed by JYCF at all of the concentrations tested $(\mathrm{P}<0.05$; Fig. 4C). The number of total entries was not significantly different between the five groups (Fig. 4D).

JYCF affects spatial learning and memory in UCMS mice. Fig. 5 shows the results of the MWM test. During the acquisition phase trials (days 1-5), the mean escape latency and search distance of $\mathrm{MC}$ mice was significantly increased compared with that in the NC group $(\mathrm{P}<0.05)$. However, compared with the MC group, mice in all JYCF groups showed a significant improvement in escape latency during the 5-days training period, and the distance in the $5 \mathrm{~g} / \mathrm{kg}$ JYCF group was decreased during the 5-day training period $(\mathrm{P}<0.05$; Fig. 5A and $\mathrm{B})$. During the probe trial, the number of platform crossings in the MC group was lower than that in the NC group and MC mice spent significantly less time in the target quadrant, which was significantly improved by JYCF at $5 \mathrm{~g} / \mathrm{kg}(\mathrm{P}<0.05$; Fig. 5C and D).

JYCF increases the synaptic density in the brains of UCMS mice. As shown in Fig. 6, synaptic density in the hippocampal neurons of MC mice was significantly decreased after 6 weeks of UCMS $(\mathrm{P}<0.01)$. Compared with the MC group, treatment with 2.5 and $5 \mathrm{~g} / \mathrm{kg} \mathrm{JYCF}$ significantly increased the synaptic density in the hippocampal neurons of UCMS-treated mice $(\mathrm{P}<0.01)$, while increases in the $1.25 \mathrm{~g} / \mathrm{kg} \mathrm{JYCF}$ group were not statistically significant $(\mathrm{P}>0.05)$.

JYCF increases the expression of synapsis-associated proteins. To further determine whether JYCF affected synapsis-associated proteins, the levels of PSD-95, Syn, BDNF and p-CREB were investigated. As shown in Fig. 7, exposure to UCMS for 6 weeks caused a significant decrease in the levels of these proteins in the hippocampi of MC mice as compared to those in the NC group, which was significantly reversed by treatment with JYCF $(2.5$ and $5 \mathrm{~g} / \mathrm{kg})$.

$J Y C F$ increases $p$-Akt and $p-G S K-3 \beta$. To elucidate the mechanism of JYCF in ameliorating the impairment of synaptic function, GSK-3 $\beta$ signaling pathways, which contribute to neuroplasticity, were studied. As shown in Fig. 8, significant decreases of $\mathrm{p}-\mathrm{Akt}(\mathrm{P}<0.05)$ and $\mathrm{p}-\mathrm{GSK}-3 \beta(\mathrm{P}<0.05)$ were observed in the hippocampi of mice in the MC and NC groups. Statistical analysis revealed that, compared with that in the MC group, JYCF treatment at $2.5 \mathrm{~g} / \mathrm{kg}$ or $5 \mathrm{~g} / \mathrm{kg}$ significantly increased the ratios of $\mathrm{p}-\mathrm{Akt} / \mathrm{AKT}$ and $\mathrm{p}-\mathrm{GSK}-3 \beta / \mathrm{GSK}-3 \beta$ compared with the MC group $(\mathrm{P}<0.05)$.

\section{Discussion}

The present study indicated, for the first time, to the best of our knowledge, that JYCF produced robust anti-depressant effects in a rodent depression model and that the underlying mechanism of this neuroprotection may be the upregulation of BDNF and p-GSK-3 $\beta$.

In the present study, administration of JYCF for 3 weeks increased the body weight of mice receiving UCMS. The preference for sucrose solution gradually recovered and other behavioral tests, such as the forced swimming, open field, plus maze and MWM test showed that regular daily administration of JYCF substantially ameliorated behavioral 

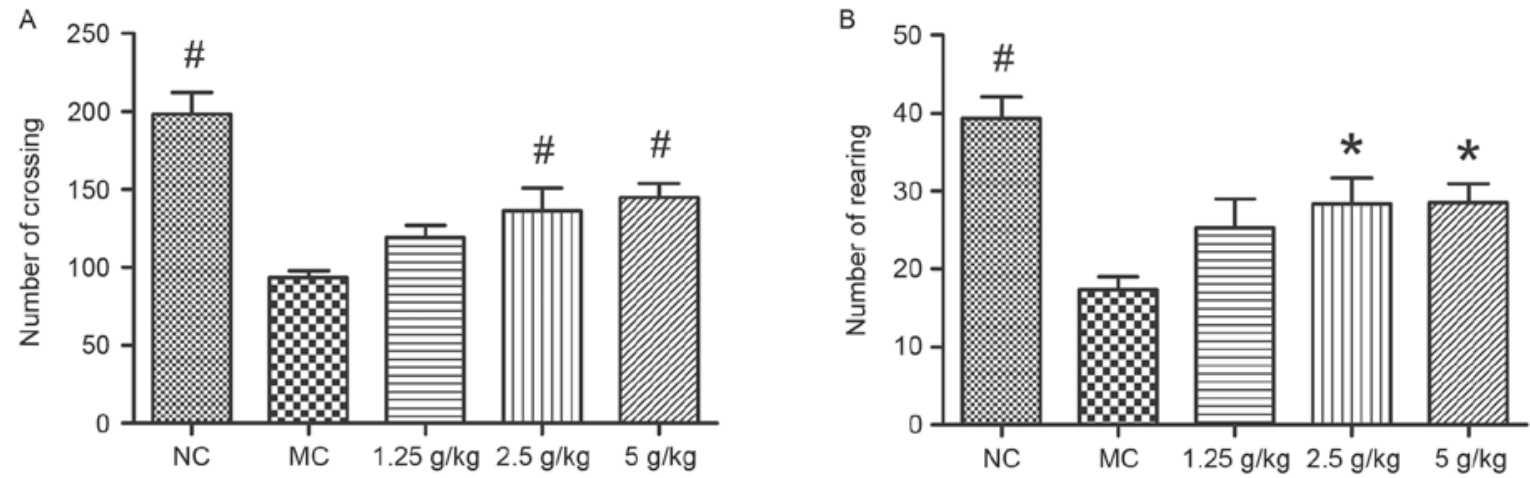

Figure 3. Results of open-field test. The number of (A) crossings and (B) rearings in NC, MC and JYCF groups was recorded. Values are expressed as the mean \pm standard error of the mean of three independent experiments. ${ }^{\mathrm{P}} \mathrm{P}<0.05,{ }^{\prime \prime} \mathrm{P}<0.01$ compared with $\mathrm{MC}$ group. NC, negative control; MC, model control group; $1.25 / 2.5 / 5 \mathrm{~g} / \mathrm{kg}$, model mice treated with $1.25 / 2.5 / 5 \mathrm{~g} / \mathrm{kg}$ JYCF; JYCF, Jieyu chufan.

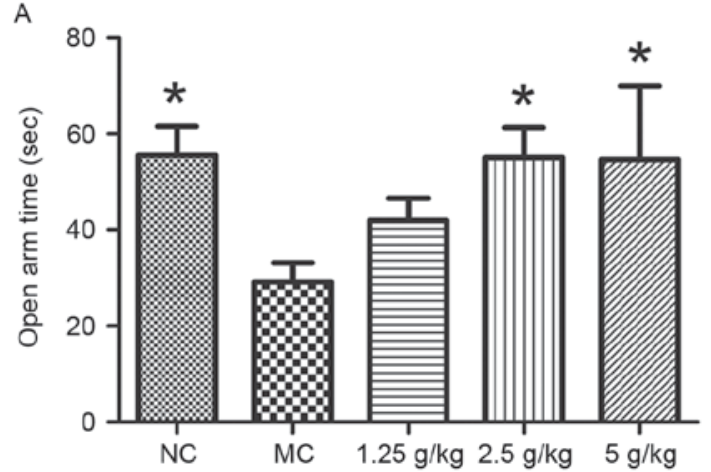

C

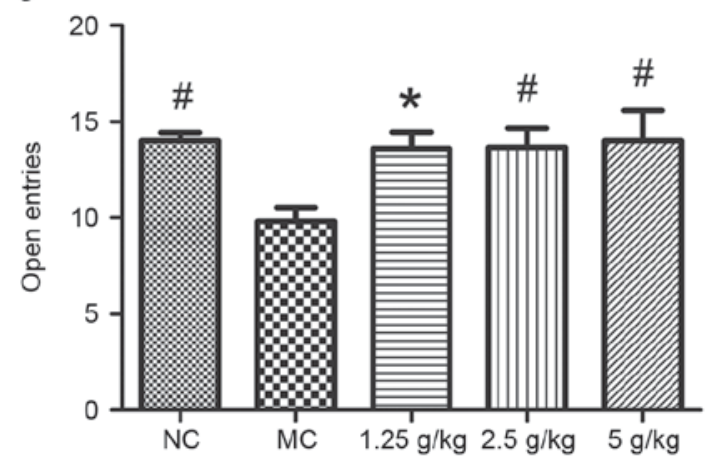

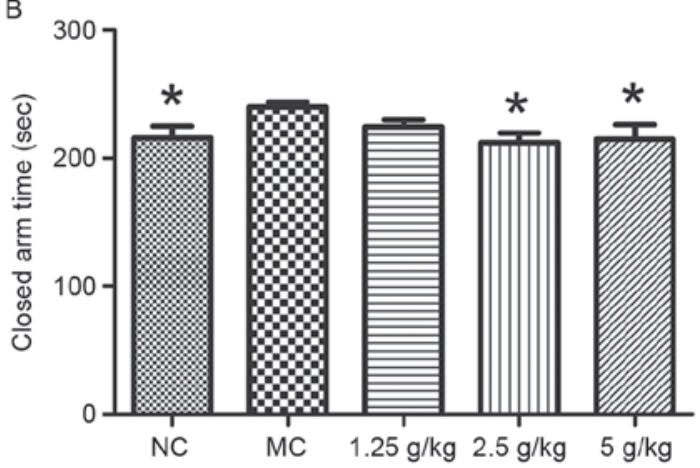

D

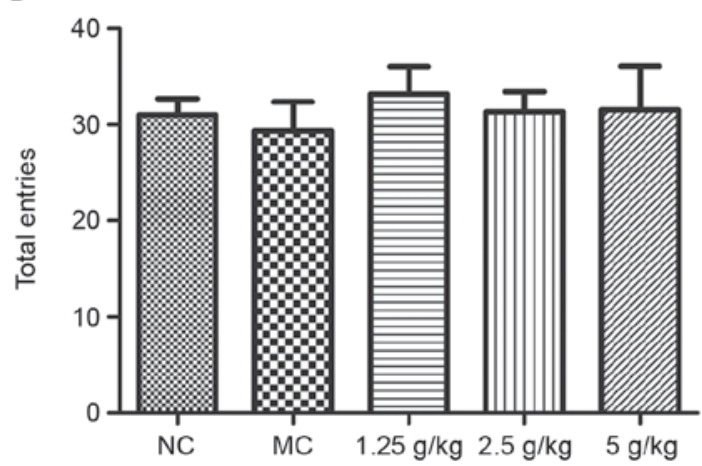

Figure 4. Results of the elevated plus maze test. (A) Time spent in the open arm, (B) time spent in the closed arm, (C) entries into the open arm and (D) total entries are shown. Total entries were similar in each group. Values are expressed as the mean \pm standard error of the mean of three independent experiments. ${ }^{*} \mathrm{P}<0.05,{ }^{\#} \mathrm{P}<0.01$ compared with MC group. NC, negative control; MC, model control group; $1.25 / 2.5 / 5 \mathrm{~g} / \mathrm{kg}$, model mice treated with $1.25 / 2.5 / 5 \mathrm{~g} / \mathrm{kg}$ Jieyu chufan.

deficits of mice subjected to UCMS. These results suggested that JYCF may be developed as a novel anti-depressant.

In spite of the vast number of studies on depression, the current understanding of the precise neurobiological pathogenic mechanisms is limited. Morphometric magnetic resonance imaging studies have reported a decrease in hippocampal volume in depression patients (32). Studies on the longitudinal course of late-life depression showed that hippocampal atrophy is associated with the severity of depression $(33,34)$. Studies in animal models of depression found that chronic stress produces atrophy and dendritic arborization of the CA3 pyramidal neuron (35). Depression or stress strongly reduce the hippocampal potential for plasticity (36). An experiment using an animal model of depression also showed that electroconvulsive shock enhances neurogenesis in the hippocampus, decreases neuronal synapses and increases dendritic spine density in various cortical and limbic structures (37).

Studies on depression and anti-depressive therapies have revealed that hippocampal neurogenesis has a key role, and certain anti-depressants block or reverse neuronal deficits in the prefrontal cortex and the hippocampus (38). Other studies found that depression reduces neuronal plasticity and 
A

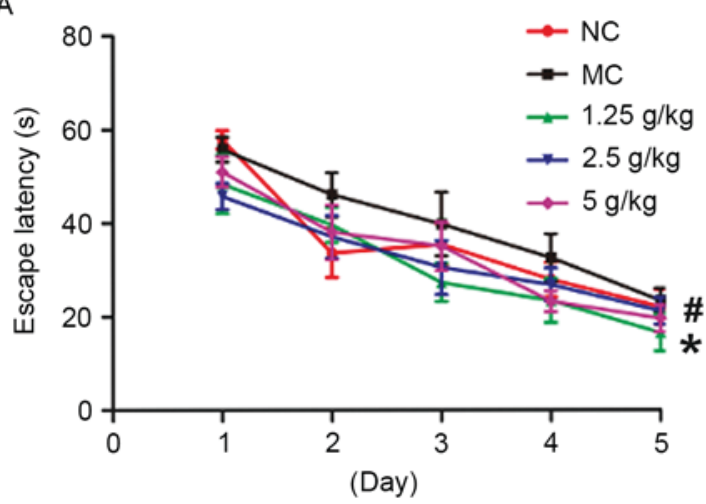

C

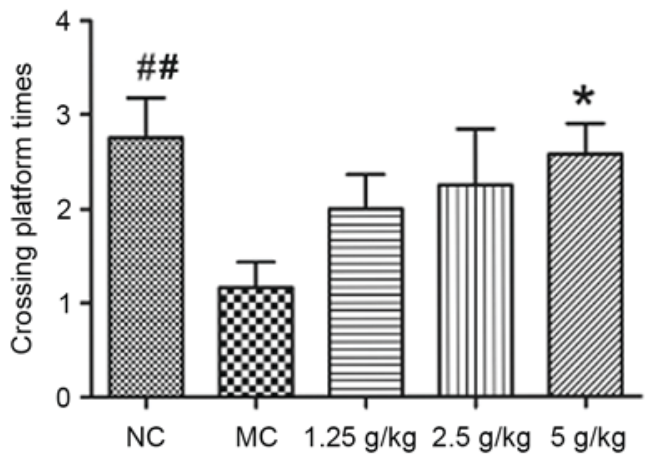

B

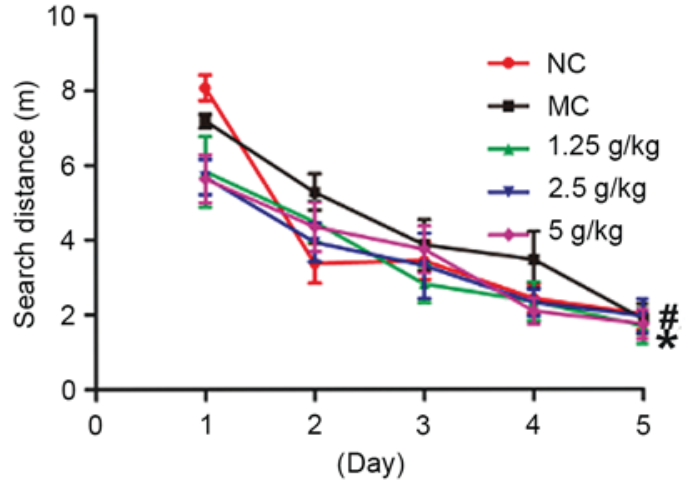

D

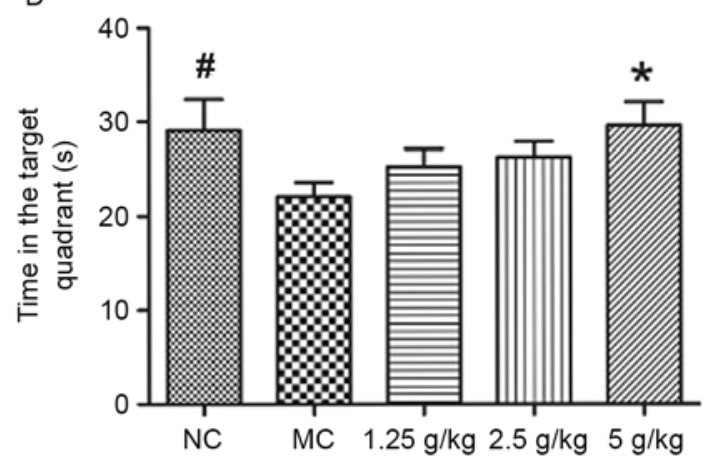

E

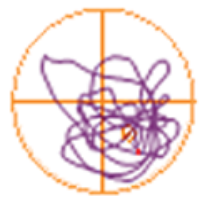

NC

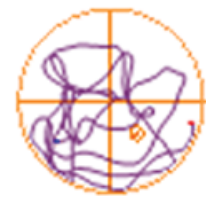

MC

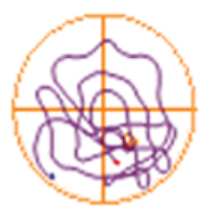

$1.25 \mathrm{~g} / \mathrm{kg}$

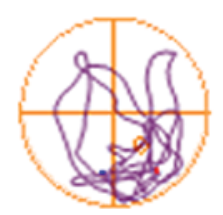

$2.5 \mathrm{~g} / \mathrm{kg}$

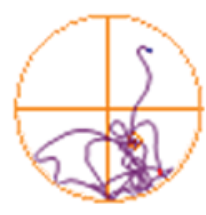

$5 \mathrm{~g} / \mathrm{kg}$

Figure 5. JYCF improves cognitive impairment in mice subjected to unpredictable chronic mild stress. (A) Escape latency for searching the submerged platform in MC group mice was significantly increased compared with the NC group, and compared with the MC group, all JYCF groups exhibited a significant decrease in escape latency. (B) Total distance for reaching the submerged platform in the MC group mice was significantly increased compared with the NC group, and the distance was decreased in the $5 \mathrm{~g} / \mathrm{kg} \mathrm{JYCF}$ group over the 5 -day training period compared with the MC group. (C) $5 \mathrm{~g} / \mathrm{kg} \mathrm{JYCF}$ treatment significantly increased the number of times the platform of MC mice in the probe trials and (D) time spent in the target quadrant, as determined in training trials. Values are expressed as the mean \pm standard error of the mean of three independent experiments. (E) Representative track plots indicating the path length to find the platform position for each group are shown. ${ }^{\#} \mathrm{P}<0.05,{ }^{\# \#} \mathrm{P}<0.01 \mathrm{NC}$ vs. MC group; ${ }^{*} \mathrm{P}<0.05,{ }^{, *} \mathrm{P}<0.01 \mathrm{JYCF}$ vs. MC group. NC, negative control; MC, model control group; $1.25 / 2.5 / 5 \mathrm{~g} / \mathrm{kg}$, model mice treated with 1.25/2.5/5 g/kg JYCF; JYCF, Jieyu chufan.

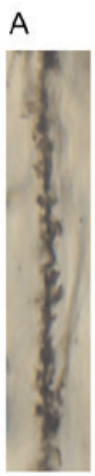

NC

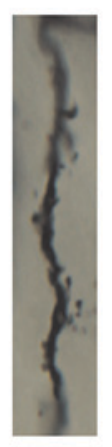

MC
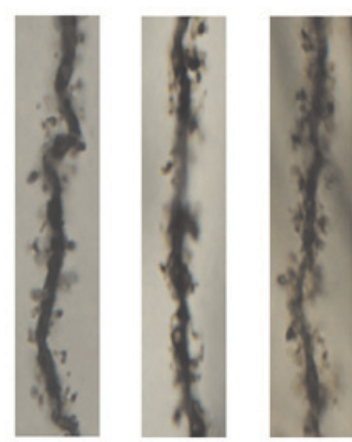

$5 \mathrm{~g} / \mathrm{kg}$
B

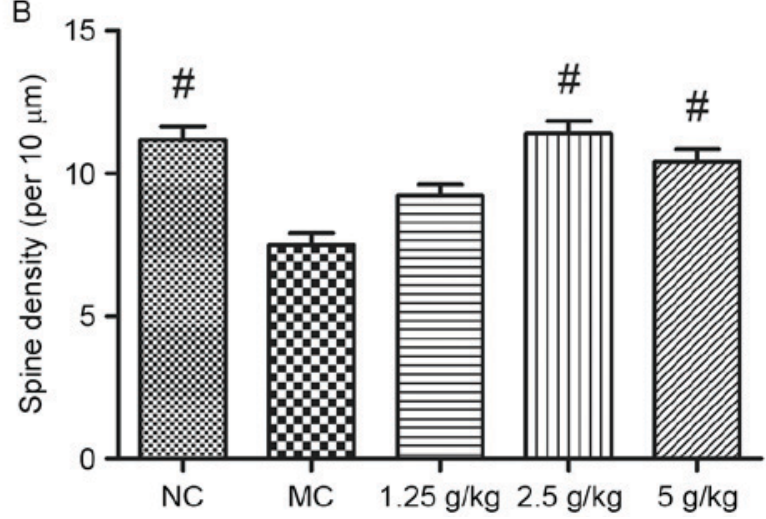

Figure 6. JYCF increases the synaptic density in mice subjected to UCMS. (A) Representative images of Golgi-Cox stained dendritic spine of the pyramidal neuron in hippocampus. (B) Bar graph showing the alteration of dendritic spine density after treatment with JYCF in mice with UCMS. Values are expressed as the mean \pm standard error of the mean of three independent experiments. ${ }^{\#} \mathrm{P}<0.01$ compared with $\mathrm{MC}$ group. NC, negative control; MC, model control group; 1.25/2.5/5 g/kg, model mice treated with 1.25/2.5/5 g/kg JYCF; JYCF, Jieyu chufan; UCMS, unpredictable chronic mild stress. 

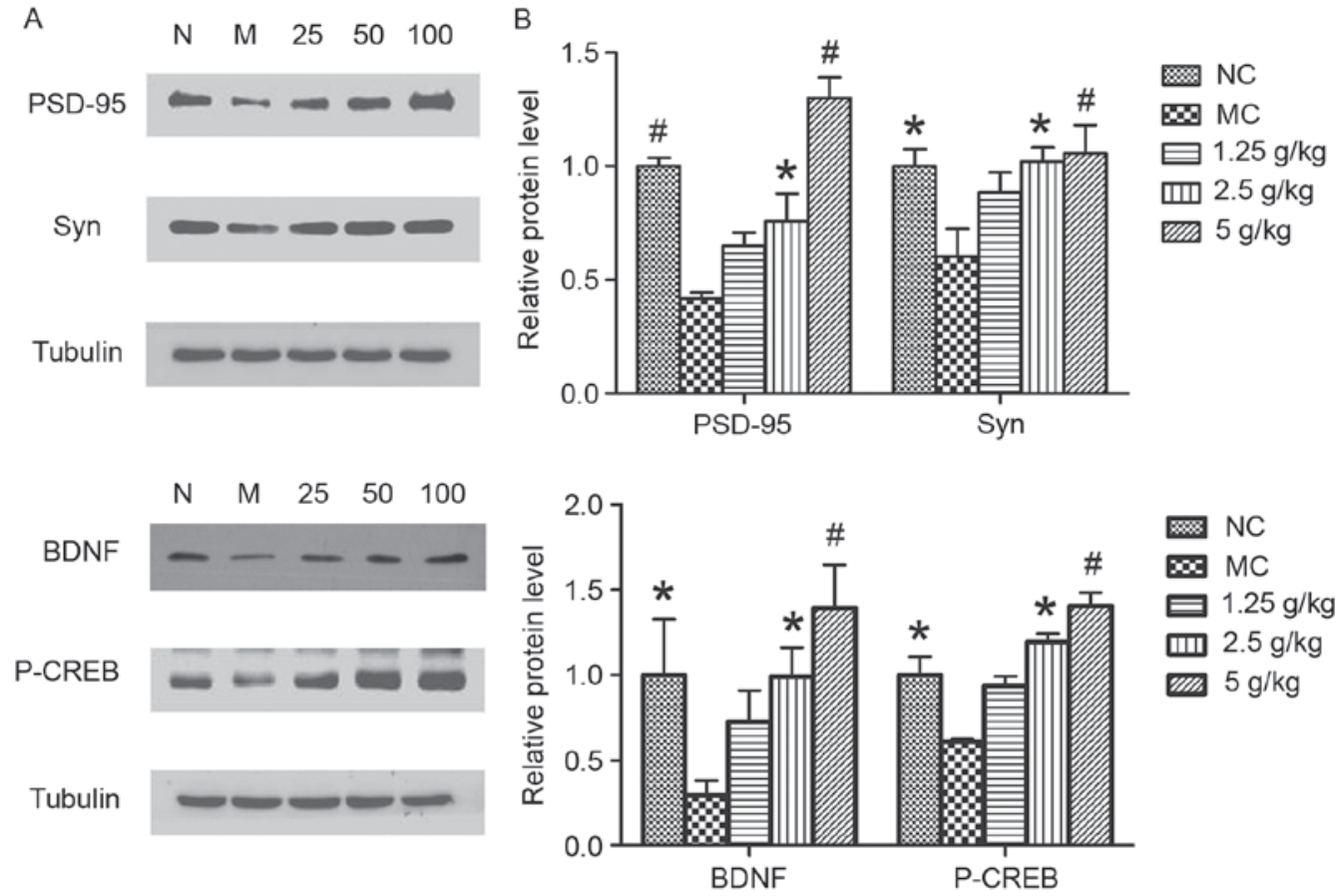

Figure 7. JYCF treatment increases the expression of synaptic-associated proteins in mice subjected to unpredictable chronic mild stress. (A) The protein levels of PSD-95, Syn, BDNF and P-CREB were determined by western blot analysis. (B) Quantitative analysis of protein expression levels. Values are expressed as the mean \pm standard error of the mean of three independent experiments. ${ }^{*} \mathrm{P}<0.05,{ }^{*} \mathrm{P}<0.01$ compared with $\mathrm{MC}$ group. PSD-95, postsynaptic density protein-95; Syn, synaptophysin; P-CREB, phosphorylated cyclic adenosine monophosphate response element binding protein; BDNF, brain-derived neurotrophic factor; GSK, glycogen synthase kinase; NC, negative control; MC, model control group; 1.25/2.5/5 g/kg, model mice treated with 1.25/2.5/5 g/kg JYCF; JYCF, Jieyu chufan.
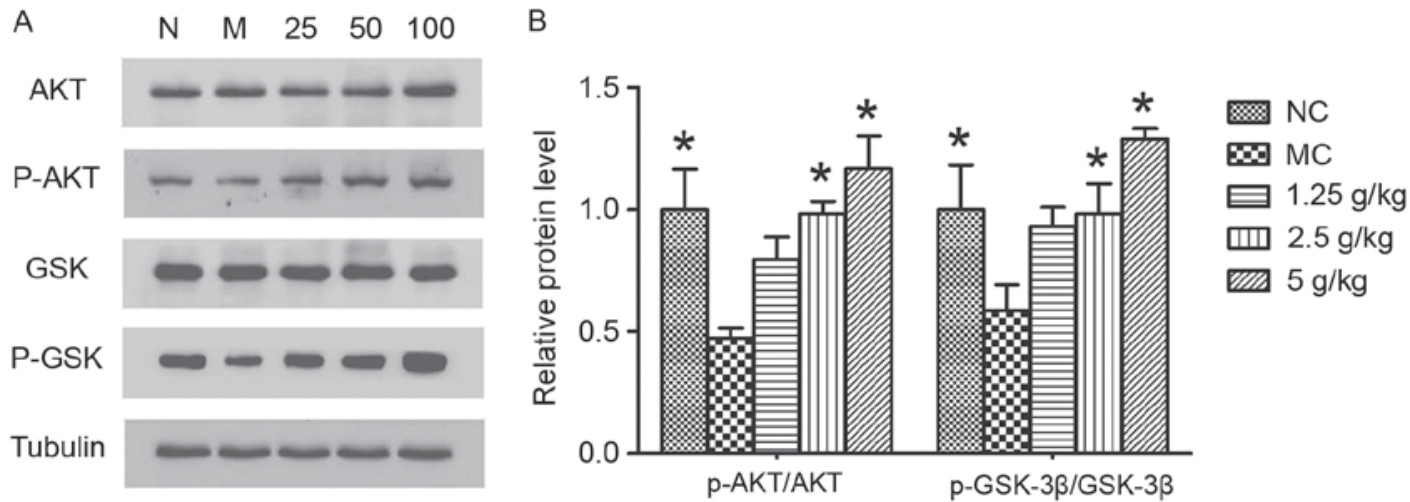

Figure 8. JYCF regulates AKT/GSK-3 $\beta$ activity in the hippocampus of mice subjected to unpredictable chronic mild stress. (A) The protein levels of AKT, P-AKT, GSK-3 $\beta$ and P-GSK-3 $\beta$ were determined by western blotting. (B) Quantitative analysis of protein expression levels was shown in p-AKT/AKT and p-GSK-3 $3 /$ GSK-3 $\beta$. Values are expressed as the mean \pm standard error of the mean of three independent experiments. " $\mathrm{P}<0.05$ compared with MC group. p-GSK, phosphorylated glycogen synthase kinase; $\mathrm{NC}$, negative control; MC, model control group; $1.25 / 2.5 / 5 \mathrm{~g} / \mathrm{kg}$, model mice treated with $1.25 / 2.5 / 5 \mathrm{~g} / \mathrm{kg}$ JYCF; JYCF, Jieyu chufan.

impairs synaptic function, which may be an important target of pharmacological intervention (39). The anti-psychotic drug Lurasidone has shown to be effective in a CMS model through the modulation of synaptic and neuroplastic proteins and PSD-95 (40). UCMS diminished the sucrose preference and reduced measures of locomotor activity in mice and decreased the expression of Syn in the hippocampal CA3 region, while electroconvulsive stimulation improved these depressive behaviors and increased the mean density of Syn (41).

In the present study, synaptic density and neurogenesis of pyramidal neurons was decreased in mice subjected to
UCMS, while JYCF administration reversed these changes and increased synaptic density and neurogenesis. Moreover, JYCF administration upregulated the expression of PSD-95 and Syn in the hippocampi of the UCMS mice, suggesting that JYCF modulates synaptic and neuroplastic proteins.

The results of the present study demonstrated that JYCF upregulated the protein levels of BDNF in the hippocampus of UCMS mice. BDNF knockout mice displayed depression-like behavior and the genetic defect regarding BDNF inhibited the effects of anti-depressants in mice exposed to UCMS (42). The interaction between CREB and BDNF is important in signal transduction and has an essential role in the concept of altered 
neuroplasticity in depression. The increase in p-CREB was significantly associated with clinical improvement in patients treated with anti-depressants or psychotherapy (43). A previous study found that the activity of CREB in peripheral blood of depression patients is increased (44). The present study showed that, the p-CREB was decreased in the MC group and treatment with JYCF was able to increase the expression of p-CREB in UCMS mice, which may be helpful in ameliorating depression.

Studies on depression-like behaviors in rodents have provided evidence that dysregulation of GSK-3 causes increased activity in specific pathways and promotes susceptibility to mood disorders (16). GSK3 was originally identified as a key enzyme of glucose metabolism and as a broadly influential enzyme that regulates a large group of transcription factors and transcriptional modulators (45). GSK-3 $\beta$, the isoform of GSK-3, is important in numerous intracellular signaling pathways of neuroplasticity (46). Overexpression of GSK-3 $\beta$ in the hippocampi of UCMS-treated mice shows a pre-depression-like behavior (47). Moreover, in CMS-treated mice or depressive patients, the expression of GSK-3 $\beta$ has been found to be significantly elevated and after treatment with GSK-3 $\beta$ inhibitors, and the depressive behavior was reversed $(48,49)$. In addition, it was reported that the phosphorylation of Akt and GSK-3 $\beta$ is a direct regulator of cell survival (50), and modulation of the activity of AKT and GSK-3 signaling may contribute to specific therapeutic effects for depression (51). In platelets of depressive patients, decreased phosphorylation of GSK-3 $\beta$ and increased GSK-3 $\beta$ activity were observed (52). The present study demonstrated decreased p-GSK-3 $\beta$ expression levels in UCMS and regular administration of JYCF increased the expression levels of phosphorylated Akt and GSK-3 $\beta$ and alleviated depression-like behaviors.

The present study revealed the antidepressant-like activity of JYCF in a chronic mild stress model and the anti-depressant-like properties mainly resulted from the modulation of synaptic structure and function and the mechanisms may be associated with the upregulation of BDNF and p-GSK-3 $\beta$ activity. The findings contributed to the understanding of the pathological effects of synaptic dysfunction in the depressive brain and suggest that JYCF may be used as a neuroprotective agent against depression.

\section{Acknowledgements}

The present study was supported by the National Science Foundation of China (grant no. 81471102), Outstanding Researcher Program of Jiangsu Province (grant no. LJ201101), the National Natural Science Foundation of Jiangsu Province of China (grant no. BK2009037), the Six Talent Peak Project of Jiangsu Province (grant no. 2015-WSN-083) and the Key Project of Nanjing Medical Science and Technology Development Project (grant no. ZKX13020).

\section{References}

1. Aldao A, Mennin DS, Linardatos E and Fresco DM: Differential patterns of physical symptoms and subjective processes in generalized anxiety disorder and unipolar depression. J Anxiety Disord 24: 250-259, 2010.
2. Trivedi MH, Rush AJ, Wisniewski SR, Nierenberg AA, Warden D, Ritz L, Norquist G, Howland RH, Lebowitz B, McGrath PJ, et al: Evaluation of outcomes with citalopram for depression using measurement-based care in STAR*D: Implications for clinical practice. Am J Psychiatry 163: 28-40, 2006.

3. Rotheneichner P, Lange S, O'Sullivan A, Marschallinger J, Zaunmair P, Geretsegger C, Aigner L and Couillard-Despres S: Hippocampal neurogenesis and antidepressive therapy: Shocking relations. Neural Plast 2014: 723915, 2014

4. Duman RS and Aghajanian GK: Synaptic dysfunction in depression: Potential therapeutic targets. Science 338: 68-72, 2012.

5. Wainwright SR and Galea LA: The neural plasticity theory of depression: Assessing the roles of adult neurogenesis and PSA-NCAM within the hippocampus. Neural Plast 2013: 805497, 2013.

6. Masi G and Brovedani P: The hippocampus, neurotrophic factors and depression: Possible implications for the pharmacotherapy of depression. CNS Drugs 25: 913-931, 2011.

7. Erickson KI, Miller DL and Roecklein KA: The aging hippocampus: Interactions between exercise, depression, and BDNF. Neuroscientist 18: 82-97, 2012

8. Yoshii A and Constantine-Paton M: Postsynaptic BDNF-TrkB signaling in synapse maturation, plasticity, and disease. Dev Neurobiol 70: 304-322, 2010.

9. Lipsky RH and Marini AM: Brain-derived neurotrophic factor in neuronal survival and behavior-related plasticity. Ann NY Acad Sci 1122: 130-143, 2007.

10. Schmidt HD and Duman RS: The role of neurotrophic factors in adult hippocampal neurogenesis, antidepressant treatments and animal models of depressive-like behavior. Behav Pharmacol 18: 391-418, 2007.

11. Castrén E, Võikar V and Rantamäki T: Role of neurotrophic factors in depression. Curr Opin Pharmacol 7: 18-21, 2007.

12. Aydemir C, Yalcin ES, Aksaray S, Kisa C, Yildirim SG, Uzbay T and Goka E: Brain-derived neurotrophic factor (BDNF) changes in the serum of depressed women. Prog Neuropsychopharmacol Biol Psychiatry 30: 1256-1260, 2006.

13. Hritcu L and Gorgan LD: Intranigral lipopolysaccharide induced anxiety and depression by altered BDNF mRNA expression in rat hippocampus. Prog Neuropsychopharmacol Biol Psychiatry 51: 126-132, 2014.

14. Musazzi L, Cattaneo A, Tardito D, Barbon A, Gennarelli M, Barlati S, Racagni G and Popoli M: Early raise of BDNF in hippocampus suggests induction of posttranscriptional mechanisms by antidepressants. BMC Neurosci 10: 48, 2009.

15. Bradley CA, Peineau S, Taghibiglou C, Nicolas CS, Whitcomb DJ, Bortolotto ZA, Kaang BK, Cho K, Wang YT and Collingridge GL: A pivotal role of GSK-3 in synaptic plasticity. Front Mol Neurosci 5: 13, 2012.

16. Liu W, Wang H, Wang Y, Li H and Ji L: Metabolic factors-triggered inflammatory response drives antidepressant effects of exercise in CUMS rats. Psychiatry Res 228: 257-264, 2015.

17. Klein PS and Melton DA: A molecular mechanism for the effect of lithium on development. Proc Natl Acad Sci USA 93: 8455-8459, 1996

18. Kaidanovich-Beilin O, Milman A, Weizman A, Pick CG and Eldar-Finkelman $\mathrm{H}$ : Rapid antidepressive-like activity of specific glycogen synthase kinase-3 inhibitor and its effect on beta-catenin in mouse hippocampus. Biol Psychiatry 55: 781-784, 2004.

19. Shapira M, Licht A, Milman A, Pick CG, Shohami E and Eldar-Finkelman H: Role of glycogen synthase kinase-3beta in early depressive behavior induced by mild traumatic brain injury. Mol Cell Neurosci 34: 571-577, 2007.

20. Lao Y, Wang X, Xu N, Zhang $\mathrm{H}$ and $\mathrm{Xu} \mathrm{H}$ : Application of proteomics to determine the mechanism of action of traditional Chinese medicine remedies. J Ethnopharmacol 155: 1-8, 2014.

21. Sun Y, Feng F and Yu X: Pharmacokinetics of geniposide in Zhi-Zi-Hou-Pu decoction and in different combinations of its constituent herbs. Phytother Res 26: 67-72, 2012.

22. Li JM, Kong LD, Wang YM, Cheng CH, Zhang WY and Tan WZ: Behavioral and biochemical studies on chronic mild stress models in rats treated with a Chinese traditional prescription Banxia-houpu decoction. Life Sci 74: 55-73, 2003.

23. Ma Z, Ji W, Qu R, Wang M, Yang W, Zhan Z, Fu Q and Ma S: Metabonomic study on the antidepressant-like effects of banxia houpu decoction and its action mechanism. Evid Based Complement Alternat Med 2013: 213739, 2013.

24. Willner P, Towell A, Sampson D, Sophokeous S and Muscat R: Reduction of sucrose preference by chronic unpredictable mild stress, and its restoration by a tricyclic antidepressant. Psychopharmacology (Berl) 93: 358-364, 1987. 
25. Grippo AJ, Beltz TG, Weiss RM and Johnson AK: The effects of chronic fluoxetine treatment on chronic mild stress-induced cardiovascular changes and anhedonia. Biol Psychiatry 59: 309-316, 2006

26. Benelli A, Filaferro M, Bertolini A and Genedani S: Influence of S-adenosyl-L-methionine on chronic mild stress-induced anhedonia in castrated rats. Br J Pharmacol 127: 645-654, 1999.

27. Forbes NF, Stewart CA, Matthews K and Reid IC: Chronic mild stress and sucrose consumption: Validity as a model of depression. Physiol Behav 60: 1481-1484, 1996.

28. Porsolt RD, Anton G, Blavet N and Jalfre M: Behavioural despair in rats: A new model sensitive to antidepressant treatments. Eur J Pharmacol 47: 379-391, 1978.

29. Zomkowski AD, Engel D, Gabilan NH and Rodrigues AL: Involvement of NMDA receptors and L-arginine-nitric oxide-cyclic guanosine monophosphate pathway in the antidepressant-like effects of escitalopram in the forced swimming test. Eur Neuropsychopharmacol 20: 793-801, 2010.

30. Iwamoto Y, Morinobu S, Takahashi T and Yamawaki S: Single prolonged stress increases contextual freezing and the expression of glycine transporter 1 and vesicle-associated membrane protein 2 mRNA in the hippocampus of rats. Prog Neuropsychopharmacol Biol Psychiatry 31: 642-651, 2007.

31. Yu L, Wang S, Chen X, Yang $\mathrm{H}$, Li X, Xu Y and Zhu X Orientin alleviates cognitive deficits and oxidative stress in A $31-42$-induced mouse model of Alzheimer's disease. Life Sci 121: 104-109, 2015.

32. Ezzati A, Zimmerman ME, Katz MJ and Lipton RB: Hippocampal correlates of depression in healthy elderly adults. Hippocampus 23: 1137-1142, 2013.

33. Taylor WD, McQuoid DR, Payne ME, Zannas AS, MacFall JR and Steffens DC: Hippocampus atrophy and the longitudinal course of late-life depression. Am J Geriatr Psychiatry 22: $1504-1512,2014$

34. André C, Dinel AL, Ferreira G, Layé $S$ and Castanon N: Diet-induced obesity progressively alters cognition, anxiety-like behavior and lipopolysaccharide-induced depressive-like behavior: Focus on brain indoleamine 2,3-dioxygenase activation. Brain Behav Immun 41: 10-21, 2014.

35. Lupien SJ, Nair NP, Brière S, Maheu F, Tu MT, Lemay M McEwen BS and Meaney MJ: Increased cortisol levels and impaired cognition in human aging: Implication for depression and dementia in later life. Rev Neurosci 10: 117-139, 1999.

36. Lucassen PJ, Stumpel MW, Wang Q and Aronica E: Decreased numbers of progenitor cells but no response to antidepressant drugs in the hippocampus of elderly depressed patients. Neuropharmacology 58: 940-949, 2010.

37. Ampuero E, Rubio FJ, Falcon R, Sandoval M, Diaz-Veliz G, Gonzalez RE, Earle N, Dagnino-Subiabre A, Aboitiz F, Orrego $\mathrm{F}$ and Wyneken U: Chronic floxetine treatment induces structural plasticity and selective changes in glutamate receptor subunits in the rat cerebral cortex. Neuroscience 169: 98-108, 2010.

38. Tanti A and Belzung C: Neurogenesis along the septo-temporal axis of the hippocampus: Are depression and the action of antidepressants region-specific? Neuroscience 252: 234-252, 2013.
39. Rayen I, Gemmel M, Pauley G, Steinbusch HW and Pawluski JL: Developmental exposure to SSRIs, in addition to maternal stress, has long-term sex-dependent effects on hippocampal plasticity. Psychopharmacology (Berl) 232: 1231-1244, 2015.

40. Luoni A, Macchi F, Papp M, Molteni R and Riva MA: Lurasidone exerts antidepressant properties in the chronic mild stress model through the regulation of synaptic and neuroplastic mechanisms in the rat prefrontal cortex. Int J Neuropsychopharmacol 18: pii: pyu061, 2014

41. Li W, Liu L, Liu YY, Luo J, Lin JY, Li X, Wang B and Min S: Effects of electroconvulsive stimulation on long-term potentiation and synaptophysin in the hippocampus of rats with depressive behavior. J ECT 28: 111-117, 2012.

42. Yulug B, Ozan E, Gönül AS and Kilic E: Brain-derived neurotrophic factor, stress and depression: A minireview. Brain Res Bull 78: 267-269, 2009.

43. Koch JM, Hinze-Selch D, Stingele K, Huchzermeier C, Goder R, Seeck-Hirschner M and Aldenhoff JB: Changes in CREB phosphorylation and BDNF plasma levels during psychotherapy of depression. Psychother Psychosom 78: 187-192, 2009.

44. Pláteník J, Fišar Z, Buchal R, Jirák R, Kitzlerová E, Zvěřová M and Raboch J: GSK-3 $\beta$, CREB, and BDNF in peripheral blood of patients with Alzheimers disease and depression. Prog Neuropsychopharmacol Biol Psychiatry 50: 83-93, 2014.

45. Jope RS: Glycogen synthase kinase-3 in the etiology and treatment of mood disorders. Front Mol Neurosci 4: 16, 2011.

46. Jope RS and Johnson GV: The glamour and gloom of glycogen synthase kinase-3. Trends Biochem Sci 29: 95-102, 2004. Wada A: Lithium and neuropsychiatric therapeutics: Neuroplasticity via glycogen synthase kinase-3beta, beta-catenin, and neurotrophin cascades. J Pharmacol Sci 110: 14-28, 2009.

47. Zhang K, Song X, Xu Y, Li X, Liu P, Sun N, Zhao X, Liu Z, Xie Z and Peng J: Continuous GSK-3 $\beta$ overexpression in the hippocampal dentate gyrus induces prodepressant-like effects and increases sensitivity to chronic mild stress in mice. J Affect Disord 146: 45-52, 2013.

48. Silva R, Mesquita AR, Bessa J, Sousa JC, Sotiropoulos I, Leão P, Almeida OF and Sousa N: Lithium blocks stress-induced changes in depressive-like behavior and hippocampal cell fate: The role of glycogen-synthase-kinase-3beta. Neuroscience 152: 656-669, 2008.

49. Oh DH, Park YC and Kim SH: Increased glycogen synthase kinase- $3 \beta$ mRNA level in the hippocampus of patients with major depression: A study using the Stanley neuropathology consortium integrative database. Psychiatry Investig 7: 202-207, 2010.

50. Nayak G and Cooper GM: p53 is a major component of the transcriptional and apoptotic program regulated by PI3-kinase/Akt/GSK3 signaling. Cell Death Dis 3: e400, 2012.

51. Kitagishi Y, Kobayashi M, Kikuta K and Matsuda S: Roles of PI3K/AKT/GSK-3/mTOR pathway in cell signaling of mental illnesses. Depress Res Treat 2012: 752563, 2012.

52. Pláteník J, Fišar Z, Buchal R, Jirák R, Kitzlerová E, Zvěřová M and Raboch J: GSK3 $\beta$, CREB, and BDNF in peripheral blood of patients with Alzheimer's disease and depression. Prog Neuropsychopharmacol Biol Psychiatry 50: 83-93, 2014. 